\title{
SELF-ADJOINT DIFFERENCE OPERATORS AND SYMMETRIC AL-SALAM-CHIHARA POLYNOMIALS
}

\author{
JACOB S. CHRISTIANSEN AND ERIK KOELINK
}

\begin{abstract}
The symmetric Al-Salam-Chihara polynomials for $q>1$ are associated with an indeterminate moment problem. There is a self-adjoint second order difference operator on $\ell^{2}(\mathbb{Z})$ to which these polynomials are eigenfunctions. We determine the spectral decomposition of this self-adjoint operator. This leads to a class of discrete orthogonality measures, which have been obtained previously by Christiansen and Ismail using a different method, and we give an explicit orthogonal basis for the corresponding weighted $\ell^{2}$-space. In particular, the orthocomplement of the polynomials is described explicitly. Taking a limit we obtain all the $N$-extremal solutions to the $q^{-1}$-Hermite moment problem, a result originally obtained by Ismail and Masson in a different way. Some applications of the results are discussed.
\end{abstract}

AMS classification: Primary 47B36; Secondary 44A60

Keywords and phrases: Difference operators; Al-Salam-Chihara polynomials; Spectral analysis; Denseness of polynomials

\section{INTRODUCTION}

The Al-Salam-Chihara polynomials were introduced originally by Al-Salam and Chihara [3] in their search for polynomials satisfying a convolution identity, see [2, §8] and also [22, Thm. 4.5] for a generalization of the convolution property of the Al-Salam-Chihara polynomials. They have been studied by Askey and Ismail [5, §3], and it turns out that they fit in the Askey-scheme of basic hypergeometric orthogonal polynomials, see [17]. In case the base $q$ is $>1$ the Al-Salam-Chihara polynomials may be related to an indeterminate moment problem. The precise conditions on the parameters for this to happen is given in [5, Thm. 3.2]. When $q>1$ and the moment problem is determinate, the orthogonality measure is purely discrete [5. (3.82)] and the dual polynomials are little $q$-Jacobi polynomials. This observation can be found at various places in the literature, see Groenevelt [14, Rmk. 3.1], Atakishiyev and Klimyk [6, §3] and Rosengren [23].

The indeterminate moment problems arising in the Askey-scheme have been classified and studied in [8]. In this paper we study the symmetric Al-Salam-Chihara polynomials for $q>1$. They are associated with an indeterminate moment problem, see [9] for more information on various solutions of the moment problem in question. In fact, they form a one-parameter extension of the continuous $q^{-1}$-Hermite polynomials, which correspond to one of the very few examples, if not the only example, of an indeterminate moment problem for which all $N$-extremal measures are known explicitly, see Ismail and Masson [16] for details. The main idea of this paper is to exploit the fact that the Al-Salam-Chihara polynomials occur in the Askey-scheme. This implies that they are eigenfunctions to a specific second order difference operator $L$. We look for a suitable Hilbert space $\mathcal{H}$ of functions such that $L$ is a self-adjoint 
operator on $\mathcal{H}$. The spectral decomposition of $L$ then gives information on denseness of polynomials in $\mathcal{H}$, or on the orthocomplement of the space of polynomials in $\mathcal{H}$. This has been exploited in our previous paper [10] for the Stieltjes-Wigert polynomials, but the case here is much simpler. See also [11] for the $q$-Laguerre polynomials and 21] for the continuous dual $q^{-1}$-Hahn polynomials, where in both cases the result occurred as a by-product of the study of a certain self-adjoint operator.

In Section 2 we introduce the symmetric Al-Salam-Chihara polynomials, and we recall some of their properties. Section 3 is the main part of the paper. We first rewrite the second order difference operator $L$ as a self-adjoint operator acting on $\ell^{2}(\mathbb{Z})$. We show that this operator is trace-class, and even in every Schatten class, by estimating its singular values. Next we list several solutions to the corresponding difference equation using known contiguous relations for basic hypergeometric series, see Lemma 3.2. With this information at hand we can give the spectral decomposition for $L$ explicitly, using standard techniques as described e.g. in [19]. It turns out that the spectrum has a positive discrete part corresponding to the symmetric AlSalam-Chihara polynomials with parameter $\beta$, and a negative discrete part corresponding to the symmetric Al-Salam-Chihara polynomials with parameter $1 / q^{2} \beta$. The special cases $\beta \downarrow 0$ and $\beta=1 / q$ are related to the continuous $q^{-1}$-Hermite polynomials. As explained in Section 4 , taking the limit $\beta \downarrow 0$ we obtain the $N$-extremal solutions to the $q^{-1}$-Hermite moment problem, a result originally due to Ismail and Masson [16]. The proof of Ismail and Masson is based on explicit descriptions of the entire functions in the Nevanlinna parametrization and heavily relies on theta function identities. We emphasize that our proof is different, but that the outcome of the $N$-extremal measures is a lucky coincidence. The case $\beta=1 / q$ is related to orthogonality measures studied by Christiansen and Ismail [9]. We prove some of their results in a different way, and we extend some of their results as well. In particular, in [9] the derivation of the measures $\lambda_{\alpha}^{(\beta)}$ is based on the use of Bailey's ${ }_{6} \psi_{6}$-summation. In the present setting, we can reverse the argument to obtain special cases of Bailey's ${ }_{6} \psi_{6}$-summation.

\section{Al-Salam-Chinara polynomials}

In this section we fix the notation for the Al-Salam-Chihara polynomials, and we recall some results for these polynomials. We use the standard notation for basic hypergeometric series as in Gasper and Rahman [12]. By switching to base $q^{-1}$, we now assume that $0<q<1$. The Al-Salam-Chihara polynomials in base $q^{-1}$ are, see e.g. [5], [17],

$$
\begin{aligned}
P_{n}\left(u ; a, b \mid q^{-1}\right) & =\frac{\left(a b ; q^{-1}\right)_{n}}{a^{n}}{ }_{3} \varphi_{2}\left(\begin{array}{c}
q^{n}, a u, a / u \\
a b, 0
\end{array} q^{-1}, q^{-1}\right) \\
& =\frac{(-b)^{n}(1 / a b ; q)_{n}}{q^{\left(\begin{array}{c}
n \\
2
\end{array}\right)}}{ }_{3} \varphi_{1}\left(\begin{array}{c}
q^{-n}, 1 / a u, u / a \\
1 / a b
\end{array} ; q, \frac{a q^{n}}{b}\right) \\
& =\frac{(-a b u)^{n}(1 / a b ; q)_{n}}{q^{\left(\begin{array}{c}
n \\
2
\end{array}\right)}}{ }_{3} \varphi_{2}\left(\begin{array}{c}
q^{-n}, 1 / a u, 1 / b u \\
1 / a b, 0
\end{array} ; q, q\right) \\
& =\frac{(-b)^{n}(u / b ; q)_{n}}{q^{\left(\begin{array}{c}
n \\
2
\end{array}\right)}}{ }_{2} \varphi_{1}\left(\begin{array}{c}
q^{-n}, 1 / a u \\
b q^{1-n} / u
\end{array} ; q, \frac{a q}{u}\right),
\end{aligned}
$$


which is a polynomial of degree $n$ in $\frac{1}{2}\left(u+u^{-1}\right)$. In (2.1) we use [12, Exercise 1.4(i)] to switch from base $q^{-1}$ to base $q$ and [12, (III.7), (III.8)] to rewrite the result as a ${ }_{3} \varphi_{2^{-}}$and ${ }_{2} \varphi_{1}$-series. Since the Al-Salam-Chihara polynomials are symmetric in $a \leftrightarrow b$ and $u \leftrightarrow u^{-1}$, we get several more expressions for them.

The Al-Salam-Chihara polynomials are eigenfunctions to a second order difference operator, which is a special case of the difference operator for the Askey-Wilson polynomials by setting two parameters equal to zero, see [12], [17, §3.8]. Explicitly, $P_{n}(u)$ as in (2.1) satisfies

$$
\left(q^{n}-1\right) P_{n}(u)=A(u)\left[P_{n}(u / q)-P_{n}(u)\right]+A\left(u^{-1}\right)\left[P_{n}(u q)-P_{n}(u)\right]
$$

with

$$
A(u)=\frac{(1-a u)(1-b u)}{\left(1-u^{2}\right)\left(1-u^{2} / q\right)} .
$$

The three-term recursion generating $P_{n}(u)$ is, see [3], [5, §3.8], [17, §3.8],

$$
\left(u+u^{-1}\right) P_{n}(u)=P_{n+1}(u)+(a+b) q^{-n} P_{n}(u)+\left(1-q^{-n}\right)\left(1-a b q^{1-n}\right) P_{n-1}(u),
$$

with initial values $P_{-1}(u)=0$ and $P_{0}(u)=1$.

In this paper we only deal with the symmetric Al-Salam-Chihara polynomials, i.e. the case $a=-b$, although we comment on the general case in Remark 3.11. By putting $u=i e^{-y}$ so that $\frac{1}{2}\left(u+u^{-1}\right)=-i \sinh y$, we define

$$
Q_{n}(y):=Q_{n}(y ; \beta \mid q)=i^{n} P_{n}\left(i e^{-y} ; \sqrt{\beta},-\sqrt{\beta} \mid q^{-1}\right)
$$

for $\beta>0$. The limiting case $\beta \rightarrow 0$ can be obtained from the explicit expression

$$
Q_{n}(y)=i^{n} \beta^{n / 2} \frac{\left(-i e^{-y} / \sqrt{\beta} ; q\right)_{n}}{q^{\left(\begin{array}{c}
n \\
2
\end{array}\right)}{ }_{2} \varphi_{1}}\left(\begin{array}{c}
q^{-n},-i e^{y} / \sqrt{\beta} \\
i e^{y} q^{1-n} \sqrt{\beta}
\end{array} ; q,-i q e^{y} \sqrt{\beta}\right)
$$

or by considering the limit transition $\beta \rightarrow 0$ in the three-term recurrence relation

$$
\left(e^{y}-e^{-y}\right) Q_{n}(y)=Q_{n+1}(y)+q^{-n}\left(1-q^{n}\right)\left(1+\beta q^{1-n}\right) Q_{n-1}(y) .
$$

We introduce the symmetric Al-Salam-Chihara polynomials as

$$
h_{n}^{(\beta)}(x \mid q)=Q_{n}(y ; \beta \mid q), \quad x=\sinh y .
$$

They satisfy the recurrence relation

$$
2 x h_{n}^{(\beta)}(x \mid q)=h_{n+1}^{(\beta)}(x \mid q)+q^{-n}\left(1-q^{n}\right)\left(1+\beta q^{1-n}\right) h_{n-1}^{(\beta)}(x \mid q),
$$

and comparing this with the three-term recursion for the continuous $q^{-1}$-Hermite polynomials, see [16, (1.16)], [9, (1.1)],

$$
2 x h_{n}(x \mid q)=h_{n+1}(x \mid q)+q^{-n}\left(1-q^{n}\right) h_{n-1}(x \mid q),
$$

we see that $\lim _{\beta \downarrow 0} h_{n}^{(\beta)}(x \mid q)=h_{n}(x \mid q)$. Moreover, we obtain $h_{n}^{(1 / q)}(x \mid q)=h_{n}\left(x \mid q^{2}\right)$ by comparing (2.6) and (2.7), cf. [9, §2]. Note that (2.6) and Favard's theorem, see e.g. [5, §2], [12, $\S 7.1]$, [19], implies that the symmetric Al-Salam-Chihara polynomials are orthogonal on $\mathbb{R}$. Moreover, by [5, Thm. 3.2] it follows that the corresponding moment problem is indeterminate. 
Combining (2.4) and (2.2) we see that

$$
\begin{aligned}
q^{n} Q_{n}(y)= & \frac{1+\beta e^{-2 y}}{\left(1+e^{-2 y}\right)\left(1+e^{-2 y} / q\right)}\left(Q_{n}(y+\ln q)-Q_{n}(y)\right) \\
& \quad+Q_{n}(y)+\frac{1+\beta e^{2 y}}{\left(1+e^{2 y}\right)\left(1+e^{2 y} / q\right)}\left(Q_{n}(y-\ln q)-Q_{n}(y)\right)
\end{aligned}
$$

and the corresponding difference operator will be the topic of Section 3 .

\section{Spectral DeComposition of A SELF-ADJoint DifFEREnCE OPERATOR}

In this section we introduce the self-adjoint operator $L$ that can be obtained from (2.8) and start out studying its general properties. Then we look for sufficiently many eigenfunctions in order to determine the spectral decomposition explicitly. As a by-product we obtain orthogonality relations involving the symmetric Al-Salam-Chihara polynomials.

3.1. Self-adjoint difference operator. Fix $\alpha \in \mathbb{R} \backslash\{0\}$ and define

$$
x_{k}(\alpha)=\frac{1}{2}\left(\frac{1}{\alpha q^{k}}-\alpha q^{k}\right), \quad k \in \mathbb{Z} .
$$

Note that $x_{k}(-\alpha)=x_{-k}(1 / \alpha)$ and that $x_{k}(\alpha) \rightarrow \pm \infty$ as $k \rightarrow \pm \infty$. We denote by $Z(\alpha)$ the grid $\left\{x_{k}(\alpha) \mid k \in \mathbb{Z}\right\}$ and have $Z(\alpha)=Z(\gamma)$ if and only if $\alpha / \gamma \in q^{\mathbb{Z}}$ or $-\alpha \gamma \in q^{\mathbb{Z}}$. So it suffices to consider $\alpha \in(q, 1]$, and $\mathbb{R}=\cup_{\alpha \in(q, 1]} Z(\alpha)$ as a union of disjoint sets. Restricting to $\alpha \in(q, 1]$ we see that $Z(\alpha)=-Z(\alpha)$ only for $\alpha=\sqrt{q}$ and $\alpha=1$.

With the above notation the eigenvalue difference equation (2.8) for $Q_{n}$ can be regarded as an operator acting on $\mathcal{F}(\alpha):=\{f: Z(\alpha) \rightarrow \mathbb{C}\}$. Defining $\delta_{l} \in \mathcal{F}(\alpha)$ by $\delta_{l}\left(x_{k}(\alpha)\right)=\delta_{k, l}$, we get a basis for the finitely supported functions in $\mathcal{F}(\alpha)$ and the operator $L=L(\alpha, \beta)$ is given by

$$
\begin{aligned}
L \delta_{l}= & \frac{1+\beta \alpha^{2} q^{2 l+2}}{\left(1+\alpha^{2} q^{2 l+2}\right)\left(1+\alpha^{2} q^{2 l+1}\right)} \delta_{l+1} \\
& +\left(1-\frac{1+\beta \alpha^{2} q^{2 l}}{\left(1+\alpha^{2} q^{2 l}\right)\left(1+\alpha^{2} q^{2 l-1}\right)}-\frac{1+\beta \alpha^{-2} q^{-2 l}}{\left(1+\alpha^{-2} q^{-2 l}\right)\left(1+\alpha^{-2} q^{-2 l-1}\right)}\right) \delta_{l} \\
& +\frac{1+\beta \alpha^{-2} q^{-2 l+2}}{\left(1+\alpha^{-2} q^{-2 l+2}\right)\left(1+\alpha^{-2} q^{-2 l+1}\right)} \delta_{l-1} .
\end{aligned}
$$

To see how (3.2) is motivated from (2.8), restrict (2.8) to the grid $Z(\alpha)$, i.e. replace $e^{-y}$ by $\alpha q^{l}$. Then identify the polynomial $Q_{n}(y)$ with the element $\sum_{l} Q_{n}\left(x_{l}(\alpha)\right) \delta_{l} \in \mathcal{F}(\alpha)$, so that $Q_{n}(y \pm \ln q)$ is identified with $\sum_{l} Q_{n}\left(x_{l \mp 1}(\alpha)\right) \delta_{l} \in \mathcal{F}(\alpha)$. Inserting this in (2.8) and shifting $l$ such that all terms involve $Q_{n}\left(x_{l}(\alpha)\right)$, we see that the right-hand side of (2.8) leads to the definition of $L$ in (3.2).

Note that (2.8) shows that the Al-Salam-Chihara polynomials are eigenfunctions to $L$ corresponding to the eigenvalues $q^{n}, n \geq 0$. We can identify $\mathcal{F}(\alpha)$ with functions on $\mathbb{Z}$ and want to consider the operator $L$ with respect to a rescaled basis $\delta_{l}=h_{l} e_{l}, h_{l} \in \mathbb{C}$, such that 
$L$ becomes a symmetric operator when considered on $\ell^{2}(\mathbb{Z})$ with the standard orthonormal basis $\left\{e_{l}\right\}_{l \in \mathbb{Z}}$. By a straightforward calculation we see that this requires

$$
\frac{h_{l+1}^{2}}{h_{l}^{2}}=\alpha^{2} q^{2 l+1} \frac{\left(\beta+\alpha^{2} q^{2 l}\right)\left(1+\alpha^{2} q^{2 l+2}\right)}{\left(1+\alpha^{2} q^{2 l}\right)\left(1+\beta \alpha^{2} q^{2 l+2}\right)},
$$

and then $L=L(\alpha, \beta)$ acts in the basis $\left\{e_{l}\right\}_{l \in \mathbb{Z}}$ by

$$
L e_{l}=a_{l} e_{l+1}+b_{l} e_{l}+a_{l-1} e_{l-1},
$$

where

$$
a_{l}=\frac{\alpha q^{l+\frac{1}{2}}}{1+\alpha^{2} q^{2 l+1}} \sqrt{\frac{\left(\beta+\alpha^{2} q^{2 l}\right)\left(1+\beta \alpha^{2} q^{2 l+2}\right)}{\left(1+\alpha^{2} q^{2 l}\right)\left(1+\alpha^{2} q^{2 l+2}\right)}}
$$

and

$$
b_{l}=1-\frac{1+\beta \alpha^{2} q^{2 l}}{\left(1+\alpha^{2} q^{2 l}\right)\left(1+\alpha^{2} q^{2 l-1}\right)}-\frac{1+\beta \alpha^{-2} q^{-2 l}}{\left(1+\alpha^{-2} q^{-2 l}\right)\left(1+\alpha^{-2} q^{-2 l-1}\right)} .
$$

It follows immediately from (3.5) that

$$
a_{l}= \begin{cases}\alpha \sqrt{\beta} q^{l+\frac{1}{2}}+\mathcal{O}\left(q^{3 l}\right), & l \rightarrow \infty, \\ \alpha^{-1} \sqrt{\beta} q^{-l-\frac{1}{2}}+\mathcal{O}\left(q^{-3 l}\right), & l \rightarrow-\infty .\end{cases}
$$

Similarly, after simplifying (3.6) to

$$
b_{l}=\frac{\alpha^{2}(1+q)(1-\beta q) q^{2 l-1}}{\left(1+\alpha^{2} q^{2 l+1}\right)\left(1+\alpha^{2} q^{2 l-1}\right)},
$$

it follows that

$$
b_{l}= \begin{cases}\alpha^{2}(1+q)(1-\beta q) q^{2 l-1}+\mathcal{O}\left(q^{4 l}\right), & l \rightarrow \infty, \\ \alpha^{-2}(1+q)(1-\beta q) q^{-2 l-1}+\mathcal{O}\left(q^{-4 l}\right), & l \rightarrow-\infty .\end{cases}
$$

Stressing the dependence of the coefficients on $\alpha$ and $\beta$, we have

$$
a_{l}\left(\alpha, 1 / \beta q^{2}\right)=\frac{a_{l}(\alpha, \beta)}{q \beta}, \quad b_{l}\left(\alpha, 1 / \beta q^{2}\right)=-\frac{b_{l}(\alpha, \beta)}{q \beta},
$$

and this implies that

$$
-q \beta L\left(\alpha, 1 / \beta q^{2}\right)=U L(\alpha, \beta) U^{*},
$$

where $U \in \mathcal{B}\left(\ell^{2}(\mathbb{Z})\right)$ is the unitary involution given by $U e_{l}=(-1)^{l} e_{l}$. Moreover, since $a_{l}(-\alpha, \beta)=-a_{l}(\alpha, \beta)$ and $b_{l}(-\alpha, \beta)=b_{l}(\alpha, \beta)$,

$$
L(-\alpha, \beta)=U L(\alpha, \beta) U^{*} .
$$

Furthermore, $a_{-l-1}(1 / \alpha, \beta)=a_{l}(\alpha, \beta)$ and $b_{-l}(1 / \alpha, \beta)=b_{l}(\alpha, \beta)$, which implies

$$
L(1 / \alpha, \beta)=V L(\alpha, \beta) V^{*}
$$

for $V \in \mathcal{B}\left(\ell^{2}(\mathbb{Z})\right)$ given by $V e_{l}=e_{-l}$.

As a consequence of (3.11), we can restrict ourselves to the case $0 \leq \beta \leq 1 / q$ (or $\beta \geq 1 / q)$ without loss of generality. Note that $b_{l}(\alpha, \beta)=0$ for all $l \in \mathbb{Z}$ precisely when $\beta=1 / q$. 
Proposition 3.1. L defined by (3.4) -(3.6) on the dense subspace $\mathcal{D} \subset \ell^{2}(\mathbb{Z})$ of finite linear combinations of elements from the orthonormal basis $\left\{e_{l}\right\}_{l \in \mathbb{Z}}$ extends to a bounded self-adjoint operator on $\ell^{2}(\mathbb{Z})$. Moreover, $L$ is a compact operator with singular values $s_{n}(L)=\mathcal{O}\left(q^{n / 2}\right)$. In particular, $L$ belongs to every Schatten $p$-class, $0<p<\infty$.

For the general theory of operator ideals we refer to Gohberg and Kreŭn [13. However, we find it more convenient to number the singular values starting from $n=0$.

Proof. From the explicit forms of $a_{l}$ and $b_{l}$ in (3.5)-(3.6) and the estimates in (3.7), (3.9) it follows that the sequences $\left\{a_{l}\right\}_{l \in \mathbb{Z}}$ and $\left\{b_{l}\right\}_{l \in \mathbb{Z}}$ are bounded, so that $L$ extends to a bounded operator on $\ell^{2}(\mathbb{Z})$. The self-adjointness of $L$ follows directly from the fact that $\left\langle L e_{l}, e_{k}\right\rangle=$ $\left\langle e_{l}, L e_{k}\right\rangle$ for all $l, k \in \mathbb{Z}$, since $a_{l}, b_{l} \in \mathbb{R}$.

To prove the second part of the proposition observe that for an operator $L$ of the form as in (3.4) the operator norm satisfies, cf. [19, Lemma 3.3.3] for the case of Jacobi operators,

$$
\|L\| \leq 2 \sup _{l \in \mathbb{Z}}\left|a_{l}\right|+2 \sup _{l \in \mathbb{Z}}\left|b_{l}\right|
$$

Define $P_{2 k+1}, k \geq 0$, as the orthogonal projection on $\operatorname{span}\left\{e_{-k}, e_{-k+1}, \ldots, e_{k}\right\}$ and $P_{2 k}, k \geq 0$, as the orthogonal projection on $\operatorname{span}\left\{e_{-k+1}, e_{-k+2}, \ldots, e_{k}\right\}$ with the convention that $P_{0}$ is the zero-projection. Then $\operatorname{dim} \operatorname{Ran}\left(P_{n}\right)=n$ for all $n \geq 0$.

First note that, by (3.14), (3.7) and (3.9),

$$
\left\|L-P_{2 k+1} L\right\| \leq 2 \sup _{l \in \mathbb{Z},|l| \geq k}\left|a_{l}\right|+2 \sup _{l \in \mathbb{Z},|l| \geq k}\left|b_{l}\right|=\mathcal{O}\left(q^{k}\right),
$$

so that $L$ can be approximated in operator norm by the finite rank operators $P_{2 k+1} L$. This implies that $L$ is a compact operator. We similarly have

$$
\left\|L-P_{2 k} L\right\| \leq 2 \sup _{l \in \mathbb{Z},|l| \geq k}\left|a_{l}\right|+2 \sup _{l \in \mathbb{Z},|l| \geq k}\left|b_{l}\right|=\mathcal{O}\left(q^{k}\right) .
$$

By [13, Thm. 2.1] the $n$-th singular value is

$$
s_{n}(L)=\min \{\|L-K\| \mid K \text { finite rank operator, } \operatorname{dim} \operatorname{Ran}(K) \leq n\}
$$

and by taking $K=P_{n} L$ we get the desired result.

3.2. Eigenfunctions for $L$. In order to derive the spectral decomposition for the self-adjoint operator $L$ we use the approach of finding the resolvent in terms of suitably behaved eigenfunctions, see e.g. [19]. So we look for solutions to

$$
z \psi_{l}(z)=a_{l} \psi_{l+1}(z)+b_{l} \psi_{l}(z)+a_{l-1} \psi_{l-1}(z), \quad l \in \mathbb{Z},
$$

and, more specifically, for $z \in \mathbb{C} \backslash \mathbb{R}$ we need to determine the subspaces $S_{z}^{ \pm}$consisting of solutions $\left\{\psi_{l}(z)\right\}_{l \in \mathbb{Z}}$ to (3.15) such that $\sum_{l=N}^{\infty}\left|\psi_{ \pm l}(z)\right|^{2}<\infty$ for some $N \in \mathbb{Z}$. Clearly $\operatorname{dim} S_{z}^{ \pm} \leq 2$, but since $S_{z}^{+} \cap S_{z}^{-}$is the deficiency space of $L$, Proposition 3.1 implies that $S_{z}^{+} \cap S_{z}^{-}=\{0\}$. We will show in Lemma 3.2 that both $S_{z}^{+}$and $S_{z}^{-}$are non-trivial, and it therefore follows that $\operatorname{dim} S_{z}^{ \pm}=1$.

Since the Al-Salam-Chihara polynomials are related to the little $q$-Jacobi polynomials, cf. the remark in the introduction, one may expect to be able to find solutions to (3.15) in terms of associated little $q$-Jacobi polynomials. These polynomials have been studied by Gupta, Ismail and Masson [15] as a limiting case of the associated big q-Jacobi polynomials. Instead of 
taking the appropriate limit in their results, we take the limit in the corresponding contiguous relations, which are attributed to Libis in [15]. This leads to contiguous relations for ${ }_{2} \varphi_{1}$-series.

We take $e=c w$ in [15, (2.5)], let $c \rightarrow 0$ and relabel to find the relation

$$
\begin{gathered}
\Psi^{-}-\left(\frac{(c-a)(c-b)}{(1-c)(q-c)} z+\frac{(q-a)(q-b)}{\left(q^{2}-c\right)(q-c)} z q+(1-z)\right) \Psi \\
+\frac{(c-a)(c-b)(1-a)(1-b)}{(1-c)^{2}(1-q c)(q-c)} z^{2} \Psi^{+}=0,
\end{gathered}
$$

where

$$
\Psi={ }_{2} \varphi_{1}\left(\begin{array}{c}
a, b \\
c
\end{array} ; q, z\right), \quad \Psi^{ \pm}={ }_{2} \varphi_{1}\left(\begin{array}{c}
a q^{ \pm 1}, b q^{ \pm 1} \\
c q^{ \pm 2}
\end{array} ; q, z\right)
$$

Another contiguous relation,

$$
\left(\frac{q(1+q)(a b-q)}{(a q-b)(a-b q)}+z\right) \phi=\frac{q(1-a)(b+q)}{(a-b)(b-a q)} \phi^{+}+\frac{q(1-b)(a+q)}{(b-a)(a-b q)} \phi^{-}
$$

where

$$
\phi={ }_{2} \varphi_{1}\left(\begin{array}{c}
a, b \\
-q
\end{array} ; q, z\right), \quad \phi^{+}={ }_{2} \varphi_{1}\left(\begin{array}{c}
a q, b / q \\
-q
\end{array} ; q, z\right), \quad \phi^{-}={ }_{2} \varphi_{1}\left(\begin{array}{c}
a / q, b q \\
-q
\end{array} ; q, z\right),
$$

can be obtained by combining identities from [12, Ex.1.10] or else be verified directly.

In the following lemma we use the notation $\theta(z)=(z, q / z ; q)_{\infty}, z \neq 0$, for the rescaled Jacobi $\theta$-function. It satisfies the relation

$$
\theta\left(z q^{l}\right)=(-z)^{-l} q^{-\left(\begin{array}{l}
l \\
2
\end{array}\right)} \theta(z)
$$

for all $l \in \mathbb{Z}$.

Lemma 3.2. (i) The functions $\psi_{l}(z)=\psi_{l}(z ; \alpha, \beta)$ defined by

$$
\psi_{l}(z)=C_{l}(\alpha, \beta) \alpha^{l} \beta^{l / 2} q^{l^{2} / 2} \frac{(1 / z ; q)_{\infty}}{z^{l}}{ }_{2} \varphi_{1}\left(\begin{array}{c}
i \alpha \sqrt{\beta} q^{l+1},-i \alpha \sqrt{\beta} q^{l+1} \\
-\alpha^{2} q^{2 l+1}
\end{array} ; q, \frac{1}{z}\right)
$$

with

$$
\frac{C_{l}(\alpha, \beta)}{\sqrt{1+\alpha^{2} q^{2 l}}}=\frac{\sqrt{\left(-\alpha^{2} / \beta,-\alpha^{2} \beta q^{2} ; q^{2}\right)_{l}}}{\left(-\alpha^{2} q ; q\right)_{2 l}}
$$

satisfy (3.15) for $|z|>1$. Moreover, each $\psi_{l}(z)$ has an analytic continuation to $\mathbb{C} \backslash\{0\}$ such that $\left\{\psi_{l}(z)\right\}_{l \in \mathbb{Z}} \in S_{z}^{+}$.

The functions $\Psi_{l}(z)=\Psi_{l}(z ; \alpha, \beta):=\psi_{-l}(z ; 1 / \alpha, \beta)$ also satisfy (3.15) for $|z|>1$, and each $\Psi_{l}(z)$ has an analytic continuation to $\mathbb{C} \backslash\{0\}$ such that $\left\{\Psi_{l}(z)\right\}_{l \in \mathbb{Z}} \in S_{z}^{-}$.

(ii) The functions $\phi_{l}(z)=\phi_{l}(z ; \alpha, \beta)$ defined by

$$
\phi_{l}(z)=B_{l}(\alpha, \beta) q^{-l / 2} \sqrt{1+\alpha^{2} q^{2 l}}{ }_{2} \varphi_{1}\left(\begin{array}{c}
i \alpha q^{l} / \sqrt{\beta},-i q^{-l} / \alpha \sqrt{\beta} \\
-q
\end{array} ; q, q z\right),
$$

where $B_{l}(\alpha, \beta)$ is a phase factor given by

$$
B_{l}(\alpha, \beta)=(-i)^{l} e^{i \arg \left(w_{l}(\alpha, \beta)\right)}, \quad w_{l}(\alpha, \beta)=(i \alpha / \sqrt{\beta},-i \alpha \sqrt{\beta} q ; q)_{l},
$$

satisfy (3.15) for $|z|<1 / q$. 
(iii) The functions in (3.19) and (3.20) are related by

$$
\psi_{l}(z ; \alpha, \beta)=K(z ; \alpha, \beta) \phi_{l}(z ; \alpha, \beta)+K(z ;-\alpha, \beta)(-1)^{l} \phi_{l}(z ;-\alpha, \beta)
$$

with

$$
K(z ; \alpha, \beta)=\frac{(-i \alpha / \sqrt{\beta}, i \alpha \sqrt{\beta} q ; q)_{\infty}}{2\left(-q,-\alpha^{2} q ; q\right)_{\infty}} \frac{\theta(i z / \alpha \sqrt{\beta})}{(-z / \beta ; q)_{\infty}} .
$$

When $\beta>1$, this gives the analytic continuation of $\psi_{l}(z)$ to $\mathbb{C} \backslash\{0\}$. Moreover,

$$
\begin{aligned}
& \theta(i \alpha z q / \sqrt{\beta})\left(-\alpha^{2} q,-i / \alpha \sqrt{\beta}, i \sqrt{\beta} q / \alpha ; q\right)_{\infty} \psi_{l}(z) \\
& +\theta(i \alpha \sqrt{\beta} / z)\left(-1 / \alpha^{2}, i \alpha / \sqrt{\beta},-i \alpha \sqrt{\beta} q ; q\right)_{\infty} \Psi_{l}(z)= \\
& \theta(1 / z) \theta\left(-1 / \alpha^{2}\right)(-q,-\beta q / z ; q)_{\infty} \phi_{l}(z) .
\end{aligned}
$$

We can use the symmetries (3.11), (3.12), and (3.13) to write down many more solutions, in particular $(-1)^{l} \phi_{l}(z ;-\alpha, \beta)$ from Lemma 3.2(iii) is a solution to (3.15). However, most of these solutions can also be obtained from standard transformation formulae for basic hypergeometric series, such as in [12, App. III].

The function $K(\cdot ; \pm \alpha, \beta)$ has an essential singularity at 0 and simple poles at $-\beta q^{-k}, k \geq 0$. So in case $\beta>1$, Lemma 3.2(iii) gives the analytic extension of $\psi_{l}(z)$ to $\mathbb{C} \backslash\{0\}$. But the limit case $\beta \downarrow 0$ is an important special case. For $l$ sufficiently large, however, the analytic extension of $\psi_{l}(z)$ to $\mathbb{C} \backslash\{0\}$ follows easily from Heine's transformation [12, (1.4.1)],

$$
\begin{aligned}
& (1 / z ; q)_{\infty 2} \varphi_{1}\left(\begin{array}{c}
i \alpha \sqrt{\beta} q^{l+1},-i \alpha \sqrt{\beta} q^{l+1} \\
-\alpha^{2} q^{2 l+1}
\end{array} ; q, \frac{1}{z}\right) \\
& =\frac{\left(-i \alpha \sqrt{\beta} q^{l+1}, i \alpha \sqrt{\beta} q^{l+1} / z ; q\right)_{\infty}}{\left(-\alpha^{2} q^{2 l+1} ; q\right)_{\infty}} \varphi_{1}\left(\begin{array}{c}
1 / z,-i \alpha q^{l} / \sqrt{\beta} \\
i \alpha \sqrt{\beta} q^{l+1} / z
\end{array} ; q,-i \alpha \sqrt{\beta} q^{l+1}\right)
\end{aligned}
$$

and the fact that $(c ; q)_{\infty 2} \varphi_{1}(a, b ; c ; q, z)$ is analytic in its parameters.

Note that the phase factor in Lemma 3.2(ii) also can be written as

$$
B_{l}(\alpha, \beta)=(-i)^{l} \sqrt{\frac{(i \alpha / \sqrt{\beta},-i \alpha \sqrt{\beta} q ; q)_{l}}{(-i \alpha / \sqrt{\beta}, i \alpha \sqrt{\beta} q ; q)_{l}}} .
$$

For future reference we write down an explicit expression for $\Psi_{l}(z)=\Psi_{l}(z ; \alpha, \beta)$, viz.

$$
\Psi_{l}(z)=\frac{(1 / z ; q)_{\infty} z^{l}}{C_{l}(\alpha, \beta) \alpha^{l} \beta^{l / 2} q^{l^{2} / 2}}{ }_{2} \varphi_{1}\left(\begin{array}{c}
i \sqrt{\beta} q^{1-l} / \alpha,-i \sqrt{\beta} q^{1-l} / \alpha \\
-q^{1-2 l} / \alpha^{2}
\end{array} ; q, \frac{1}{z}\right) .
$$

Proof. The fact that the functions $\psi_{l}(z ; \alpha, \beta)$ for $|z|>1$ and $\phi_{l}(z ; \alpha, \beta)$ for $|z|<1 / q$ satisfy (3.15) follows directly from (3.16) and (3.17). Since $\psi_{l}(z)$ and $\phi_{l}(z)$ are analytic, their analytic extensions satisfy (3.15) as well. Invoking (3.13) shows that $\Psi_{l}(z)$ also satisfies (3.15).

The first relation in (iii) follows from Heine's transformation formula [12, (1.4.3)] and the formula [12, (4.3.2)] for the analytic continuation of a ${ }_{2} \varphi_{1}$-series. The second relation follows from [12, (3.3.5)], using [12, (1.4.5)] for the second ${ }_{2} \varphi_{1}$-series and (3.18).

The explicit expression of $\psi_{l}(z)$ in (3.19) shows immediately that $\left\{\psi_{l}(z)\right\} \in S_{z}^{+}$for $|z|>1$ due to the factor $q^{l^{2} / 2}$. For the general case we use (3.22) to see that $\psi_{l}(z)$ behaves like

$$
C(\alpha, \beta) z^{-l} \alpha^{l} \beta^{l / 2} q^{l^{2} / 2} \text { as } l \rightarrow \infty,
$$


where $C(\alpha, \beta)=\lim _{l \rightarrow \infty} C_{l}(\alpha, \beta)$. Once we have the analytic continuation of $\psi_{l}(z)$ to $\mathbb{C} \backslash\{0\}$ for some large value of $l$, we can get the analytic continuation for other values of $l$ by determining $\psi_{l}(z)$ recursively from (3.15).

The statement for $\Psi_{l}(z)$ follows similarly.

Remark 3.3. Note that ${ }_{2} \varphi_{1}$-series similar to the ones in Lemma 3.2 already have occurred in the spectral decomposition of certain self-adjoint operators. Only the parameter regimes are somewhat different. This is of great influence on the particular structure of the spectral decomposition. See [18] for the case of the curly exponential $\mathcal{E}_{q}(z ; t)$ related to a not essentially self-adjoint operator. Another related case concerns little $q$-Jacobi functions and the AskeyWilson $q$-Hankel transform as discussed in [20].

3.3. Spectral decomposition of $L$. The resolvent of $L$ can be written in terms of solutions in $S_{z}^{ \pm}$and their Wronskian, see (3.26) below. Recall that the Wronskian of two sequences $\phi=\left\{\phi_{l}\right\}_{l \in \mathbb{Z}}$ and $\psi=\{\psi\}_{l \in \mathbb{Z}}$ is the sequence defined by $[\phi, \psi]_{l}=a_{l}\left(\phi_{l+1} \psi_{l}-\phi_{l} \psi_{l+1}\right), l \in \mathbb{Z}$. It is well-known that $[\phi, \psi]_{l}$ is independent of $l$ in case both $\psi$ and $\phi$ satisfy (3.15), see e.g. [19] for more information.

Proposition 3.4. The Wronskian of $\psi(z)=\left\{\psi_{l}(z)\right\}_{l \in \mathbb{Z}}$ and $\Psi(z)=\left\{\Psi_{l}(z)\right\}_{l \in \mathbb{Z}}$ is given by

$$
[\psi(z), \Psi(z)]=-z(-q \beta / z, 1 / z ; q)_{\infty}, \quad z \neq 0 .
$$

The proof of the proposition is based on the following lemma.

Lemma 3.5. Assume that $\beta \leq 1 / q$. As $x \downarrow 0$, we have

$$
{ }_{2} \varphi_{1}\left(\begin{array}{c}
i q \sqrt{\beta} x,-i q \sqrt{\beta} x \\
-x^{2} q
\end{array} ; q, 1 / z\right) \rightarrow \frac{1}{(1 / z ; q)_{\infty}}
$$

and

$$
{ }_{2} \varphi_{1}\left(\begin{array}{c}
i q \sqrt{\beta} / x,-i q \sqrt{\beta} / x \\
-q / x^{2}
\end{array} ; q, 1 / z\right) \rightarrow(-\beta q / z ; q)_{\infty} .
$$

The convergence is uniform for $z$ in compact subsets of $|z|>1$.

Proof. The ${ }_{2} \varphi_{1}$-series can be written as

$$
\sum_{n=0}^{\infty} \frac{(i q \sqrt{\beta} x,-i q \sqrt{\beta} x ; q)_{n}}{\left(-x^{2} q, q ; q\right)_{n}}(1 / z)^{n}=1+\sum_{n=1}^{\infty} \frac{\left(1+x^{2} \beta q^{2}\right) \cdots\left(1+x^{2} \beta q^{2 n}\right)}{\left(1+x^{2} q\right) \cdots\left(1+x^{2} q^{n}\right)} \frac{(1 / z)^{n}}{(q ; q)_{n}}
$$

respectively

$$
\sum_{n=0}^{\infty} \frac{(i q \sqrt{\beta} / x,-i q \sqrt{\beta} / x ; q)_{n}}{\left(-q / x^{2}, q ; q\right)_{n}}(1 / z)^{n}=1+\sum_{n=1}^{\infty} \frac{\left(x^{2}+\beta q^{2}\right) \cdots\left(x^{2}+\beta q^{2 n}\right)}{\left(x^{2}+q\right) \cdots\left(x^{2}+q^{n}\right)} \frac{(1 / z)^{n}}{(q ; q)_{n}},
$$

using the fact that $(a,-a ; q)_{n}=\left(a^{2} ; q^{2}\right)_{n}$. So the termwise convergence is clear recalling Euler's power series expansions of the $q$-exponential functions, see [12, (1.3.15-16)]. When $\beta \leq 1 / q$ we have $\beta q^{j} \leq 1$ for all $j \geq 1$, and the result follows by dominated convergence. The statement on uniform convergence in $|z|>1$ is straightforward.

Note that the proof easily can be adapted to the case $\beta \leq q^{-k}, k \in \mathbb{N}$ fixed. 
Proof of Proposition 3.4. Assuming that $|z|>1$ it follows from Lemma 3.5 and (3.19), (3.23) that, as $l \rightarrow \infty$,

$$
\psi_{l}(z) \sim C_{l}(\alpha, \beta) \alpha^{l} \beta^{l / 2} q^{l^{2} / 2} z^{-l} \quad \text { and } \quad \Psi_{l}(z) \sim \frac{z^{l}(1 / z,-q \beta / z ; q)_{\infty}}{C_{l}(\alpha, \beta) \alpha^{l} \beta^{l / 2} q^{l^{2} / 2}} .
$$

Combining this with the asymptotic behavior of $a_{l}$ from (3.7) and an easy limit for $C_{l}(\alpha, \beta)$ as $l \rightarrow \infty$, we get the desired expression for the Wronskian. By analytic continuation and Lemma 3.2, (3.24) remains valid for $z \in \mathbb{C} \backslash\{0\}$.

We are now in position to determine the spectrum of the compact operator $L$ defined in (3.4) -(3.6) . Our considerations will also lead to an explicit orthogonal basis for $\ell^{2}(\mathbb{Z})$ consisting of eigenvectors of $L$.

The spectral measure $E$ for $L$ can be obtained from the resolvents via the formula

$$
\langle E((a, b)) v, w\rangle=\lim _{\delta \downarrow 0} \lim _{\varepsilon \downarrow 0} \frac{1}{2 \pi i} \int_{a+\delta}^{b-\delta}\left\langle(L-s-i \varepsilon)^{-1} v, w\right\rangle-\left\langle(L-s+i \varepsilon)^{-1} v, w\right\rangle d s,
$$

valid for all $v, w \in \ell^{2}(\mathbb{Z})$, see e.g. [19]. The inner products appearing in the integral can be written as

$$
\left\langle(L-(s \pm i \varepsilon))^{-1} v, w\right\rangle=\sum_{k \leq j} \frac{\psi_{j}(s \pm i \varepsilon) \Psi_{k}(s \pm i \varepsilon)}{[\psi(s \pm i \varepsilon), \Psi(s \pm i \varepsilon)]}\left(v_{k} \bar{w}_{j}+v_{j} \bar{w}_{k}\right)\left(1-\frac{1}{2} \delta_{j, k}\right)
$$

and since both $\psi_{l}(z)$ and $\Psi_{l}(z)$ are analytic in $\mathbb{C} \backslash\{0\}, E$ is concentrated on the zeros of the Wronskian $[\psi(z), \Psi(z)]$, excluding $z=0$. This is consistent with Proposition 3.1 .

Theorem 3.6. The spectrum of $L$ is given by $\sigma(L)=-\beta q^{\mathbb{N}} \cup\{0\} \cup q^{\mathbb{N}_{0}}$. The accumulation point 0 is not an eigenvalue of $L$.

Proof. The first statement follows from Proposition 3.4. To prove that 0 does not belong to the point spectrum of $L$ we use Lemma 3.2(ii), which tells us that

$$
f_{l}=B_{l}(\alpha, \beta) q^{-l / 2} \sqrt{1+\alpha^{2} q^{2 l}}, \quad l \in \mathbb{Z}
$$

is a solution to (3.15) for $z=0$. Using the symmetry (3.12), we see that

$$
g_{l}=(-1)^{l} B_{l}(-\alpha, \beta) q^{-l / 2} \sqrt{1+\alpha^{2} q^{2 l}}, \quad l \in \mathbb{Z}
$$

is yet another solution. It is clear that neither $f=\left\{f_{l}\right\}_{l \in \mathbb{Z}}$ nor $g=\left\{g_{l}\right\}_{l \in \mathbb{Z}}$ is square summable. So it suffices to show that they are linearly independent. A straightforward calculation using (3.7) shows that

$$
a_{l}\left(f_{l+1} g_{l}-f_{l} g_{l+1}\right) \rightarrow-2 i \alpha \sqrt{\beta} \quad \text { as } l \rightarrow \infty .
$$

Therefore, $[f, g]=-2 i \alpha \sqrt{\beta}$ and the linear independence follows whenever $\beta>0$.

Remark 3.7. In the study of $L$ we have sometimes assumed that $\beta>0$. Let us briefly remark on the case $\beta=0$, showing that the results in Proposition 3.4 and Theorem 3.6 remain valid after taking the limit $\beta \downarrow 0$.

The solutions in Lemma 3.2(i) can still be defined when $\beta=0$. Using the fact that

$$
\lim _{\beta \downarrow 0} C_{l}(\alpha, \beta) \beta^{l / 2}=\frac{\alpha^{l} q^{\left(\begin{array}{l}
l \\
2
\end{array}\right)} \sqrt{1+\alpha^{2} q^{2 l}}}{\left(-\alpha^{2} q ; q\right)_{2 l}}=: \frac{C_{l}(\alpha)}{\alpha^{l} q^{l^{2} / 2}}
$$


and the transformation formula

$$
(z ; q)_{\infty 2} \varphi_{1}(0,0 ; c ; q, z)={ }_{0} \varphi_{1}(-; c ; q, c z),
$$

which is a limiting case of Heine's transformation formula [12, (1.4.3)], we see that

$$
\psi_{l}(z ; \alpha, 0)=\frac{C_{l}(\alpha)}{z^{l}}{ }_{0} \varphi_{1}\left(\begin{array}{c}
- \\
-\alpha^{2} q^{2 l+1}
\end{array} ; q,-\frac{\alpha^{2} q^{2 l+1}}{z}\right)
$$

and

$$
\Psi_{l}(z ; \alpha, 0)=\frac{z^{l}}{C_{l}(\alpha)}{ }^{0} \varphi_{1}\left(\begin{array}{c}
- \\
-q^{1-2 l} / \alpha^{2}
\end{array} ; q,-\frac{q^{1-2 l}}{\alpha^{2} z}\right) .
$$

These two solutions span $S_{z}^{ \pm}$, respectively. As regards their Wronskian we observe that

$$
{ }_{0} \varphi_{1}(-; 1 / x ; q ; z / x) \rightarrow(z ; q)_{\infty} \quad \text { as } x \downarrow 0,
$$

so that the ${ }_{0} \varphi_{1}$-series in $(3.28)$ tends to $(1 / z ; q)_{\infty}$ as $l \rightarrow \infty$. Using this and the fact that

$$
a_{l}(\alpha, 0) \sim \alpha^{2} q^{2 l+1 / 2} \quad \text { as } l \rightarrow \infty,
$$

we get, in accordance with Proposition 3.4 , that the Wronskian is given by

$$
[\psi(z ; \alpha), \Psi(z ; \alpha)]=-z(1 / z ; q)_{\infty}, \quad z \neq 0 .
$$

The first part of Theorem 3.6 thus remains valid, but the proof that 0 is not an eigenvalue breaks down since the Wronskian vanishes as $\beta \downarrow 0$. This can be fixed in the following way, however. Take $z=0$ and $\beta=0$ in (3.15) using (3.5), (3.8) and multiply by

$$
\sqrt{1+\alpha^{2} q^{2 l}}\left(1+\alpha^{2} q^{2 l+1}\right)\left(1+\alpha^{2} q^{2 l-1}\right) / \alpha^{2} q^{2 l-1 / 2}
$$

to get

$$
0=\frac{q+\alpha^{2} q^{2 l}}{\sqrt{1+\alpha^{2} q^{2 l+2}}} \psi_{l+1}+\left(q^{\frac{1}{2}}+q^{-\frac{1}{2}}\right) \sqrt{1+\alpha^{2} q^{2 l}} \psi_{l}+\frac{q^{-1}+\alpha^{2} q^{2 l}}{\sqrt{1+\alpha^{2} q^{2 l-2}}} \psi_{l-1}, \quad l \in \mathbb{Z} .
$$

We are looking for two linearly independent solutions to (3.29) that do not belong to $\ell^{2}(\mathbb{Z})$. Setting $\phi_{l}=(-1)^{l} \psi_{l} / \sqrt{1+\alpha^{2} q^{2 l}}$, (3.29) reduces to

$$
0=\left(q+\alpha^{2} q^{2 l}\right) \phi_{l+1}-\left(q^{\frac{1}{2}}+q^{-\frac{1}{2}}\right)\left(1+\alpha^{2} q^{2 l}\right) \phi_{l}+\left(q^{-1}+\alpha^{2} q^{2 l}\right) \phi_{l-1}, \quad l \in \mathbb{Z}
$$

and one immediately comes up with the solution $\phi_{l}=q^{-l / 2}$. Another solution is given by

$$
\tilde{\phi}_{l}=\left(1+\alpha^{2} q^{l}\right)\left(1-q^{l}\right) q^{-3 l / 2}
$$

and whereas $\phi_{l}$ never vanishes, we have $\tilde{\phi}_{0}=0$. The two solutions, and the corresponding solutions to (3.29), are therefore linearly independent and obviously give non-square summable eigenfunctions. 
3.4. Orthogonality relations. Theorem 3.6 shows that the eigenvalues of $L$ form two sequences, namely $q^{n}, n \geq 0$, and $-\beta q^{n+1}, n \geq 0$. In the special case $\beta=0$ all the negative eigenvalues disappear. The corresponding eigenfunctions, $\psi\left(q^{n}\right)$ and $\psi\left(-\beta q^{n+1}\right)$, form an orthogonal basis for $\ell^{2}(\mathbb{Z})$ since $L$ is self-adjoint. Note that we can replace $\psi$ by $\Psi$ as the sequences are proportional. The explicit relations are as follows.

Lemma 3.8. For $n \geq 0$, we have

$$
(-1)^{n} \alpha^{2 n+2} \Psi_{l}\left(q^{n}\right)=\frac{\left(-\alpha^{2} q ; q\right)_{\infty}}{\left(-1 / \alpha^{2} ; q\right)_{\infty}} \frac{\left(-\beta q^{2} / \alpha^{2} ; q^{2}\right)_{\infty}}{\left(-\alpha^{2} \beta q^{2} ; q^{2}\right)_{\infty}} \psi_{l}\left(q^{n}\right)
$$

and

$$
(-1)^{n} \alpha^{2 n+2} \Psi_{l}\left(-\beta q^{n+1}\right)=\frac{\left(-\alpha^{2} q ; q\right)_{\infty}}{\left(-1 / \alpha^{2} ; q\right)_{\infty}} \frac{\left(-1 / \alpha^{2} \beta ; q^{2}\right)_{\infty}}{\left(-\alpha^{2} / \beta ; q^{2}\right)_{\infty}} \psi_{l}\left(-\beta q^{n+1}\right) .
$$

Proof. If we set $z=q^{n}$ or $z=-\beta q^{n+1}$ in (3.21), the right-hand side vanishes. Using (3.18) and simplifying gives the result.

As we will see below the eigenfunctions are closely related to the symmetric Al-SalamChihara polynomials. In particular, the fact that the eigenfunctions are orthogonal can be translated into orthogonality relations for the polynomials in (2.5).

Applying [12, (1.4.1)] to the ${ }_{2} \varphi_{1}$-series in (3.19) we see that $\psi_{l}\left(q^{n}\right)$ can be written as, see (2.4) and (2.5),

$$
\frac{\left(-\alpha^{2} q ; q\right)_{\infty}}{\left(-\alpha^{2} \beta q^{2} ; q^{2}\right)_{\infty}} \psi_{l}\left(q^{n}\right)=\sqrt{\frac{\left(-\alpha^{2} / \beta ; q^{2}\right)_{l}}{\left(-\alpha^{2} \beta q^{2} ; q^{2}\right)_{l}}} \alpha^{l} \beta^{l / 2} q^{l^{2} / 2} \sqrt{1+\alpha^{2} q^{2 l}}(-1)^{n} \alpha^{n} h_{n}^{(\beta)}\left(x_{l}(\alpha) \mid q\right) .
$$

Similarly, but this time applying [12, (1.4.5)] to the ${ }_{2} \varphi_{1}$-series in (3.19), we get

$$
\frac{\left(-\alpha^{2} q ; q\right)_{\infty}}{\left(-\alpha^{2} / \beta ; q^{2}\right)_{\infty}} \psi_{l}\left(-\beta q^{n+1}\right)=\sqrt{\frac{\left(-\alpha^{2} \beta q^{2} ; q^{2}\right)_{l}}{\left(-\alpha^{2} / \beta ; q^{2}\right)_{l}}} \frac{(-1)^{l} \alpha^{l} q^{l^{2} / 2}}{\beta^{l / 2} q^{l}} \sqrt{1+\alpha^{2} q^{2 l}}(-1)^{n} \alpha^{n} h_{n}^{\left(1 / \beta q^{2}\right)}\left(x_{l}(\alpha) \mid q\right) \text {. }
$$

The fact that $\left\langle\psi\left(q^{n}\right), \psi\left(-\beta q^{m+1}\right)\right\rangle=0$ for $n, m \geq 0$ therefore reads

$$
\sum_{l=-\infty}^{\infty}(-1)^{l} \alpha^{2 l}\left(1+\alpha^{2} q^{2 l}\right) q^{l(l-1)} h_{n}^{(\beta)}\left(x_{l}(\alpha) \mid q\right) h_{m}^{\left(1 / \beta q^{2}\right)}\left(x_{l}(\alpha) \mid q\right)=0
$$

A direct proof of (3.30) can also be given since

$$
\sum_{l=-\infty}^{\infty}(-1)^{l} \alpha^{2 l}\left(1+\alpha^{2} q^{2 l}\right) q^{l(l-1)} x_{l}^{k}(\alpha)=0, \quad k \geq 0,
$$

using Jacobi's triple product [12, (1.6.1)] and the binomial expansion.

In order to write down the relation $\left\langle\psi\left(q^{n}\right), \psi\left(q^{m}\right)\right\rangle=\delta_{n, m}\left\|\psi\left(q^{n}\right)\right\|^{2}$ explicitly, we need to determine the $\ell^{2}(\mathbb{Z})$-norm of the sequence $\psi\left(q^{n}\right)=\left\{\psi_{l}\left(q^{n}\right)\right\}_{l \in \mathbb{Z}}$. This will be an easy task once we know the spectral projection $E\left(\left\{q^{n}\right\}\right)$ given by, cf. (3.25),

$$
\left\langle E\left(\left\{q^{n}\right\}\right) v, w\right\rangle=-\frac{1}{2 \pi i} \oint_{\left(q^{n}\right)}\left\langle(L-s)^{-1} v, w\right\rangle d s, \quad v, w \in \ell^{2}(\mathbb{Z}) .
$$


Alluding to (3.26) and the fact that

$$
\frac{1}{2 \pi i} \oint_{\left(q^{n}\right)} \frac{\psi_{j}(s) \Psi_{k}(s)}{[\psi(s), \Psi(s)]} d s=\psi_{j}\left(q^{n}\right) \Psi_{k}\left(q^{n}\right) \operatorname{Res}_{z=q^{n}} \frac{1}{[\psi(z), \Psi(z)]},
$$

it follows from Lemma 3.8 that

$$
\left\langle E\left(\left\{q^{n}\right\}\right) v, w\right\rangle=\frac{q^{n^{2}}\left\langle v, \psi\left(q^{n}\right)\right\rangle\left\langle\psi\left(q^{n}\right), w\right\rangle}{\alpha^{2 n+2} \beta^{n}(-1 / \beta, q ; q)_{n}} \frac{\left(-\alpha^{2} q ; q\right)_{\infty}\left(-\beta q^{2} / \alpha^{2} ; q^{2}\right)_{\infty}}{\left(-1 / \alpha^{2},-\beta q, q ; q\right)_{\infty}\left(-\alpha^{2} \beta q^{2} ; q^{2}\right)_{\infty}}
$$

since

$$
\operatorname{Res}_{z=q^{n}} \frac{1}{[\psi(z), \Psi(z)]}=\frac{(-1)^{n+1} q^{n^{2}}}{\beta^{n}(-1 / \beta, q ; q)_{n}} \frac{1}{(-\beta q, q ; q)_{\infty}} .
$$

In particular, setting $v=w=\psi\left(q^{n}\right)$ we see that

$$
\left\|\psi\left(q^{n}\right)\right\|^{2}=\frac{\alpha^{2 n+2} \beta^{n}(-1 / \beta, q ; q)_{n}}{q^{n^{2}}} \frac{\left(-1 / \alpha^{2},-\beta q, q ; q\right)_{\infty}\left(-\alpha^{2} \beta q^{2} ; q^{2}\right)_{\infty}}{\left(-\alpha^{2} q ; q\right)_{\infty}\left(-\beta q^{2} / \alpha^{2} ; q^{2}\right)_{\infty}}
$$

and the orthogonality relation $\left\langle\psi\left(q^{n}\right), \psi\left(q^{m}\right)\right\rangle=\delta_{n, m}\left\|\psi\left(q^{n}\right)\right\|^{2}$ reads

$$
\begin{aligned}
\sum_{l=-\infty}^{\infty} \frac{\left(-\alpha^{2} / \beta ; q^{2}\right)_{l}}{\left(-\alpha^{2} \beta q^{2} ; q^{2}\right)_{l}} \alpha^{2 l} \beta^{l}(1 & \left.+\alpha^{2} q^{2 l}\right) q^{l^{2}} h_{n}^{(\beta)}\left(x_{l}(\alpha) \mid q\right) h_{m}^{(\beta)}\left(x_{l}(\alpha) \mid q\right) \\
& =\delta_{n, m} \frac{\beta^{n}(-1 / \beta, q ; q)_{n}}{q^{n^{2}}} \frac{\left(-\alpha^{2},-q / \alpha^{2},-\beta q, q ; q\right)_{\infty}}{\left(-\alpha^{2} \beta q^{2},-\beta q^{2} / \alpha^{2} ; q^{2}\right)_{\infty}}
\end{aligned}
$$

It follows from (3.31) that the spectral projection $E\left(\left\{q^{n}\right\}\right)$ has rank 1. In other words, the eigenvalues $q^{n}, n \geq 0$, are simple.

A similar computation can be carried out to determine the $\ell^{2}(\mathbb{Z})$-norm of $\psi\left(-\beta q^{n+1}\right)$, but the relation $\left\langle\psi\left(-\beta q^{n+1}\right), \psi\left(-\beta q^{m+1}\right)\right\rangle=\delta_{n, m}\left\|\psi\left(-\beta q^{n+1}\right)\right\|^{2}$ corresponds to (3.32) with $\beta$ replaced by $1 / \beta q^{2}$.

The orthogonality relation (3.32) was obtained by Christiansen and Ismail in [9, Thm. 6.1] using the attachment procedure combining moments with generating functions and mimicking the construction of the $N$-extremal solutions to the $q^{-1}$-Hermite moment problem. The present analysis, however, gives a much more complete picture of the situation. In particular, we obtain an explicit orthogonal basis for $L^{2}\left(\lambda_{\alpha}^{(\beta)}\right)$, where $\lambda_{\alpha}^{(\beta)}$ denotes the discrete probability measure in (3.32).

Theorem 3.9. The polynomials are dense in $L^{2}\left(\lambda_{\alpha}^{(\beta)}\right)$ if and only if $\beta=0$. When $\beta=0$, the polynomials $\left\{h_{n}(x \mid q)\right\}_{n \geq 0}$ form an orthogonal basis. For $\beta>0$, let $\Phi^{(\beta)}(x \mid q)$ denote the function given by

$$
\Phi^{(\beta)}(\sinh y \mid q)=\frac{\left(-e^{-2 y} / \beta ; q^{2}\right)_{\infty}}{\left(-e^{-2 y} \beta q^{2} ; q^{2}\right)_{\infty}} e^{-y\left(\frac{i \pi-\log \beta}{\log q}-1\right)}, \quad y \in \mathbb{R} .
$$

Then $\left\{h_{n}^{(\beta)}(x \mid q)\right\}_{n \geq 0} \cup\left\{\Phi^{(\beta)}(x \mid q) h_{n}^{\left(1 / \beta q^{2}\right)}(x \mid q)\right\}_{n \geq 0}$ form an orthogonal basis for $L^{2}\left(\lambda_{\alpha}^{(\beta)}\right)$.

Note that $\left|\Phi^{\left(1 / \beta q^{2}\right)}\right|=\left|1 / \Phi^{(\beta)}\right|$ for $\beta>0$. 
Proof. Rewrite (3.30) in terms of the orthogonality measure $\lambda_{\alpha}^{(\beta)}$ to see that

$$
(-1)^{l} \beta^{-l} q^{-l} \frac{\left(-\alpha^{2} \beta q^{2} ; q^{2}\right)_{l}}{\left(-\alpha^{2} / \beta ; q^{2}\right)_{l}} h_{m}^{\left(1 / \beta q^{2}\right)}\left(x_{l}(\alpha) \mid q\right)
$$

are orthogonal to the symmetric Al-Salam-Chihara polynomials. Moreover, by (3.32) with $\beta$ replaced by $1 / q^{2} \beta$, we see that these functions are orthogonal with respect to the measure $\lambda_{\alpha}^{(\beta)}$. Then combine the fact that

$$
\frac{\left(-\alpha^{2} \beta q^{2} ; q^{2}\right)_{l}}{\left(-\alpha^{2} / \beta ; q^{2}\right)_{l}}=\frac{\left(-\alpha^{2} \beta q^{2} ; q^{2}\right)_{\infty}}{\left(-\alpha^{2} / \beta ; q^{2}\right)_{\infty}} \frac{\left(-\alpha^{2} q^{2 l} / \beta ; q^{2}\right)_{\infty}}{\left(-\alpha^{2} \beta q^{2 l+2} ; q^{2}\right)_{\infty}}
$$

with the identity

$$
q^{l\left(\frac{i \pi-\log \beta}{\log q}-1\right)}=(-1)^{l} \beta^{-l} q^{-l}
$$

and recall that $x_{l}(\alpha)$ can be written as $\sinh y$ with $e^{y}=1 / \alpha q^{l}$.

Recalling that a solution $\mu$ to an indeterminate moment problem is $N$-extremal if and only if the polynomials $\mathbb{C}[x]$ are dense in $L^{2}(\mathbb{R}, \mu)$, see e.g. [1, Chapter 2], we have the following consequence of the above theorem.

Corollary 3.10. For $\beta>0$ the measures $\lambda_{\alpha}^{(\beta)}, \alpha \in(q, 1]$, are mutually different, non $N$ extremal solutions to the moment problem associated with the symmetric Al-Salam-Chihara polynomials.

Christiansen and Ismail [9, §7] proved this result for $\alpha=\beta=1$ in a different way. Note that our approach even tells that the measure $\lambda_{\alpha}^{(\beta)}$ cannot be canonical of any order, see e.g. [1] for more information on canonical measures. This follows from the fact that the closure of the polynomials has codimension $+\infty$ in $L^{2}\left(\lambda_{\alpha}^{(\beta)}\right)$.

Remark 3.11. In [8] a classification of the indeterminate moment problems within the $q$ analogue of the Askey scheme is given. Since each of the corresponding orthogonal polynomials also satisfies a second order difference equation, it is natural to look for a corresponding selfadjoint operator. This is done for the Stieltjes-Wigert polynomials in [10], and indirectly also in 11] for the $q$-Laguerre polynomials. It seems that for the Al-Salam-Chihara polynomials as in (2.1) we need at least $a+b \in i \mathbb{R}$ and $a b \in \mathbb{R}$, which are fulfilled for $a=-b \in \mathbb{R}$. However, in general it seems difficult to find a suitable Hilbert space of functions on which the second order difference operator can be realized as a self-adjoint operator, and some external guidance for such a choice seems necessary. See also 21 for an example involving a different type of Hilbert space related to the continuous dual $q^{-1}$-Hahn polynomials, and more generally [20] for an overview.

Remark 3.12. Since Theorem 3.9 gives an explicit orthogonal basis for the space $L^{2}\left(\lambda_{\alpha}^{(\beta)}\right)$, we also have the dual orthogonality relations

$$
\sum_{n=0}^{\infty} \frac{\psi_{l}\left(q^{n}\right) \psi_{k}\left(q^{n}\right)}{\left\|\psi\left(q^{n}\right)\right\|^{2}}+\sum_{n=0}^{\infty} \frac{\psi_{l}\left(-\beta q^{n+1}\right) \psi_{k}\left(-\beta q^{n+1}\right)}{\left\|\psi\left(-\beta q^{n+1}\right)\right\|^{2}}=\delta_{k, l} \quad \text { for } k, l \in \mathbb{Z} .
$$


Each of the series can be summed as a special case of the bilinear generating function 9 , Thm. 7.2], and we find an identity involving four balanced ${ }_{4} \varphi_{3}$-series. We do not work out the details.

Remark 3.13. The operator $L$ can also be considered as an operator acting on $L^{2}(\mathbb{R})$, and this case can be reduced to a direct integral of the cases studied in Theorems 3.6 and 3.9 since $\cup_{\alpha \in(q, 1]} Z(\alpha)=\mathbb{R}$. Because the spectrum of $L(\alpha, \beta)$ is independent of $\alpha$, this gives no new results. We merely see that the polynomials $h_{n}^{(\beta)}(x \mid q)$ are orthogonal with respect to the weight function

$$
w(x)=\frac{1}{\left(-e^{2 y} / \beta,-e^{-2 y} / \beta ; q^{2}\right)_{\infty}}, \quad x=\sinh y \in \mathbb{R},
$$

a result contained in [9, Thm. 5.1]. Observe namely that

$$
w\left(x_{l}(\alpha)\right)=\frac{\left(-\alpha^{2} / \beta ; q^{2}\right)_{l}}{\left(-\alpha^{2} \beta q^{2} ; q^{2}\right)_{l}} \frac{\alpha^{2 l} \beta^{l} q^{l(l+1)}}{\left(-1 / \alpha^{2} \beta,-\alpha^{2} / \beta ; q^{2}\right)_{\infty}}
$$

so that

$$
\lambda_{\alpha}^{(\beta)}\left(\left\{x_{l}(\alpha)\right\}\right)=M(\alpha, \beta) w\left(x_{l}(\alpha)\right) \sqrt{x_{l}^{2}(\alpha)+1}
$$

for some constant $M(\alpha, \beta)$ not depending on $l$. The factor $\sqrt{x_{l}^{2}(\alpha)+1}$ comes from the change of variables $x=\sinh y$.

See [10] for direct integral techniques applied to the case of the Stieltjes-Wigert polynomials.

\section{Special CASEs AND ADditional Results}

Continuous $q^{-1}$-Hermite polynomials. Theorem 3.9 in case $\beta=0$ is dealing with the continuous $q^{-1}$-Hermite polynomials, $h_{n}^{(0)}(x \mid q)=h_{n}(x \mid q)$. These polynomials were introduced by Askey [4] and the associated indeterminate moment problem was studied by Ismail and Masson [16] in much detail. In particular, Ismail and Masson were able to find all of the $N$-extremal solutions explicitly using the Nevanlinna parametrization. Corollary 4.1 below is thus a result due to Ismail and Masson [16, §6], but we give a different proof based on Theorem 3.9.

Corollary 4.1. The measures $\lambda_{\alpha}^{(0)}, \alpha \in(q, 1]$, constitute all of the $N$-extremal solutions to the $q^{-1}$-Hermite moment problem.

Proof. Theorem 3.9 shows that the polynomials are dense in $L^{2}\left(\lambda_{\alpha}^{(0)}\right)$ and since this characterizes the $N$-extremal measures, it follows that each $\lambda_{\alpha}^{(0)}$ is $N$-extremal. Now $N$-extremal measures are discrete and for each $x \in \mathbb{R}$ there is a unique $N$-extremal measure, say $\rho$, with $\rho(\{x\})>0$, see e.g. [1]. Given $x \in \mathbb{R}$, pick $\alpha \in(q, 1]$ such that $x=x_{l}(\alpha)$ for some $l \in \mathbb{Z}$. Then $\lambda_{\alpha}^{(0)}(\{x\})>0$ and we have obtained all of the $N$-extremal measures.

Note that the explicit orthogonality relation for the continuous $q^{-1}$-Hermite polynomials with respect to the measure $\lambda_{\alpha}^{(0)}$ is

$$
\sum_{l=-\infty}^{\infty} \alpha^{4 l}\left(1+\alpha^{2} q^{2 l}\right) q^{l(2 l-1)} h_{n}\left(x_{l}(\alpha) \mid q\right) h_{m}\left(x_{l}(\alpha) \mid q\right)=\delta_{n, m} \frac{(q ; q)_{n}}{q^{\left(\begin{array}{c}
n+1 \\
2
\end{array}\right)}}\left(-\alpha^{2},-q / \alpha^{2}, q ; q\right)_{\infty}
$$


In the special case $\beta=1 / q$, the fixed point under the involution $\beta \mapsto 1 / q^{2} \beta$, we are also dealing with the continuous $q^{-1}$-Hermite polynomials since $h_{n}^{(1 / q)}(x \mid q)=h_{n}\left(x \mid q^{2}\right)$ as observed in Section 2, In this case the eigenvectors corresponding to the eigenvalues $q^{n}$ and $-q^{n}$ differ only by a sign,

$$
\psi_{l}\left(q^{n} ; \alpha, 1 / q\right)=\alpha^{l} q^{\left(\begin{array}{l}
l \\
2
\end{array}\right)} \sqrt{1+\alpha^{2} q^{2 l}}(-1)^{n} \alpha^{n} h_{n}^{(1 / q)}\left(x_{l}(\alpha) \mid q\right)=(-1)^{l} \psi_{l}\left(-q^{n} ; \alpha, 1 / q\right) .
$$

The orthogonality relations (3.32) and (3.30) then take the simpler forms

$$
\begin{aligned}
\sum_{l=-\infty}^{\infty} \alpha^{2 l}\left(1+\alpha^{2} q^{2 l}\right) q^{l(l-1)} h_{n}^{(1 / q)} & \left(x_{l}(\alpha) \mid q\right) h_{m}^{(1 / q)}\left(x_{l}(\alpha) \mid q\right) \\
& =2 \delta_{n, m} \frac{\left(q^{2} ; q^{2}\right)_{n}}{q^{n(n+1)}}\left(-\alpha^{2},-q^{2} / \alpha^{2}, q^{2} ; q^{2}\right)_{\infty}
\end{aligned}
$$

and

$$
\sum_{l=-\infty}^{\infty}(-1)^{l} \alpha^{2 l}\left(1+\alpha^{2} q^{2 l}\right) q^{l(l-1)} h_{n}^{(1 / q)}\left(x_{l}(\alpha) \mid q\right) h_{m}^{(1 / q)}\left(x_{l}(\alpha) \mid q\right)=0 .
$$

Adding (4.3) and (4.4) leaves only the even terms in the sum, and since $x_{2 l}(\alpha)$ in base $q$ equals $x_{l}(\alpha)$ in base $q^{2}$, we obtain (4.1) in base $q^{2}$. Similarly, subtracting (4.4) from (4.3) leaves only the odd terms and we find (4.1) in base $q^{2}$ with $\alpha$ replaced by $\alpha q$.

This can be interpreted in the operator theoretic approach as follows. We have $b_{l}(\alpha, 1 / q)=0$ and it thus follows that $L^{2}$ leaves the subspaces $M^{e}$ spanned by $\left\{e_{2 l}\right\}_{l \in \mathbb{Z}}$ and $M^{o}$ spanned by $\left\{e_{2 l+1}\right\}_{l \in \mathbb{Z}}$ invariant. Stressing the dependence on $\alpha, \beta$, and $q$, it is an easy verification that

$$
\left.L(\alpha, 1 / q \mid q)^{2}\right|_{M^{e}} \simeq L\left(\alpha, 0 \mid q^{2}\right) \text { and }\left.L(\alpha, 1 / q \mid q)^{2}\right|_{M^{o}} \simeq L\left(\alpha q, 0 \mid q^{2}\right),
$$

using the fact that $M^{e} \simeq \ell^{2}(\mathbb{Z}) \simeq M^{o}$ as subspaces of $\ell^{2}(\mathbb{Z})$. Therefore,

$$
L(\alpha, 1 / q \mid q)^{2} \simeq L\left(\alpha, 0 \mid q^{2}\right) \oplus L\left(\alpha q, 0 \mid q^{2}\right) .
$$

By Theorem 3.6 the point spectrum of $L(\alpha, 1 / q \mid q)^{2}$ equals $q^{2 \mathbb{N}_{0}}$, each point having multiplicity 2. Also, by Theorem [3.6, $L\left(\alpha, 0 \mid q^{2}\right)$ and $L\left(\alpha q, 0 \mid q^{2}\right)$ have spectrum $q^{2 \mathbb{N}_{0}}$, with each point of multiplicity 1. Since we have the eigenvectors explicitly, and because of (4.2), we obtain

$$
\psi\left(q^{n} ; \alpha, 1 / q \mid q\right)+\psi\left(-q^{n} ; \alpha, 1 / q \mid q\right)=2\left(-\alpha^{2} q^{2} ; q^{2}\right)_{\infty} \psi\left(q^{2 n} ; \alpha, 0 \mid q^{2}\right)
$$

and

$$
\psi\left(q^{n} ; \alpha, 1 / q \mid q\right)-\psi\left(-q^{n} ; \alpha, 1 / q \mid q\right)=2 \alpha q^{-n}\left(-\alpha^{2} q^{4} ; q^{2}\right)_{\infty} \psi\left(q^{2 n} ; \alpha q, 0 \mid q^{2}\right),
$$

stressing the dependence on the base as well. So the measure $\lambda_{\alpha}^{(1 / q)}$ splits as a convex linear combination of two $N$-extremal measures, as observed by Christiansen and Ismail [9, §6]. In particular, the measure in question is not $N$-extremal, and Theorem 3.9 and its preceding proof shows that the orthocomplement of the polynomials is spanned by the orthogonal functions

$$
h_{n}\left(x \mid q^{2}\right) e^{-i \pi y / \log q}, \quad n \geq 0,
$$

where $x=\sinh y$. 
Summation formulae. Recall the generating function, see [7], [9],

$$
\sum_{n=0}^{\infty} h_{n}^{(\beta)}(x \mid q) \frac{q^{\left(\begin{array}{l}
n \\
2
\end{array}\right)} t^{n}}{(q ; q)_{n}}=\frac{\left(t e^{-y},-t e^{-y} ; q^{2}\right)_{\infty}}{\left(-t^{2} \beta ; q\right)_{\infty}}, \quad|t|<\frac{1}{\sqrt{\beta}} .
$$

This and Bailey's ${ }_{6} \psi_{6}$-summation formula, see [12, (5.3.1)], was used by Christiansen and Ismail [9, §6] to obtain the measures $\lambda_{\alpha}^{(\beta)}$ as solutions to the moment problem associated with the symmetric Al-Salam-Chihara polynomials. Now that we have obtained the measures $\lambda_{\alpha}^{(\beta)}$ as such solutions in a different way, we can reverse the line of reasoning and obtain a 4-parameter subfamily of Bailey's ${ }_{6} \psi_{6}$-summation formula from (4.5). Multiply (3.32) by $q^{n(n-1) / 2} t_{1}^{n} /(q ; q)_{n}$ and $q^{m(m-1) / 2} t_{2}^{m} /(q ; q)_{m}$ and sum over $n$ and $m$ using (4.5) twice. Interchange the order of summation and simplify to get

$$
\begin{aligned}
{ }_{6} \psi_{6}\left(\begin{array}{c}
i \alpha q,-i \alpha q, i \alpha / \sqrt{\beta},-i \alpha / \sqrt{\beta},-\alpha q / t_{1},-\alpha q / t_{2} \\
i \alpha,-i \alpha, i \alpha \sqrt{\beta} q,-i \alpha \sqrt{\beta} q, t_{1} \alpha, t_{2} \alpha
\end{array} ;, \frac{t_{1} t_{2} \beta}{q}\right)= \\
\frac{\left(i t_{1} \sqrt{\beta},-i t_{1} \sqrt{\beta}, i t_{2} \sqrt{\beta},-i t_{2} \sqrt{\beta},-t_{1} t_{2} / q,-\alpha^{2} q,-q / \alpha^{2},-\beta q, q ; q\right)_{\infty}}{\left(t_{1} \alpha, t_{2} \alpha,-t_{1} / \alpha,-t_{2} / \alpha, t_{1} t_{2} \beta / q, i \alpha \sqrt{\beta} q,-i \alpha \sqrt{\beta} q, i \sqrt{\beta} q / \alpha,-i \sqrt{\beta} q / \alpha ; q\right)_{\infty}}
\end{aligned}
$$

which is a special case of Bailey's ${ }_{6} \psi_{6}$-summation formula that involves 5 degrees of freedom.

In case we use (3.30) instead of (3.32), we get the summation formula

$$
{ }_{4} \psi_{4}\left(\begin{array}{c}
i \alpha q,-i \alpha q,-\alpha q / t_{1},-\alpha q / t_{2} \\
i \alpha,-i \alpha, \alpha t_{1}, \alpha t_{2}
\end{array} ; q,-\frac{t_{1} t_{2}}{q^{2}}\right)=0 .
$$

This is the special case $b=q a^{1 / 2}$ of the ${ }_{4} \psi_{4}$-summation formula [12, (5.3.3)], which is a special case of Bailey's ${ }_{6} \psi_{6}$-sum.

We note that one can extend this method by considering suitable bilinear generating functions as [9, Thm. 7.2].

\section{REFERENCES}

[1] N. I. Akhiezer, The classical moment problem and some related questions in analysis, Hafner Publishing Co., New York, 1965.

[2] W.A. Al-Salam, Characterization theorems for orthogonal polynomials, p. 1-24 in Orthogonal Polynomials (ed. P. Nevai), NATO Adv. Sci. Inst. Ser. C Math. Phys. Sci. 294, Kluwer, 1990.

[3] W.A. Al-Salam and T.S. Chihara, Convolutions of orthonormal polynomials, SIAM J. Math. Anal. 7 (1976), 16-28.

[4] R. Askey, Continuous q-Hermite polynomials when $q>1$, p. 151-158 in q-series and partitions (ed. D. Stanton), IMA Vol. Math. Appl. 18, Springer, 1989.

[5] R. Askey and M. Ismail, Recurrence relations, continued fractions, and orthogonal polynomials, Mem. Amer. Math. Soc. 49 (1984), no. 300.

[6] N.M. Atakishiyev and A.U. Klimyk, On q-orthogonal polynomials, dual to little and big q-Jacobi polynomials, J. Math. Anal. Appl. 294 (2004), 246-257.

[7] T.S. Chihara and M.E.H. Ismail, Extremal measures for a system of orthogonal polynomials, Constr. Approx. 9 (1993), 111-119.

[8] J.S. Christiansen, Indeterminate moment problems within the Askey-scheme, Ph.D. thesis, University of Copenhagen (2004), http://www.math.ku.dk/ stordal/thesis.pdf

[9] J.S. Christiansen and M.E.H. Ismail, A moment problem and a family of integral evaluations, Trans. Amer. Math. Soc. 358 (2006), 4071-4097. 
[10] J.S. Christiansen and E. Koelink, Self-adjoint difference operators and classical solutions to the StieltjesWigert moment problem, J. Approx. Theory 140 (2006), 1-26.

[11] N. Ciccoli, E. Koelink and T.H. Koornwinder, q-Laguerre polynomials and big q-Bessel functions and their orthogonality relations, Methods Appl. Anal. 6 (1999), 109-127.

[12] G. Gasper and M. Rahman, Basic Hypergeometric Series, 2nd ed., Cambridge Univ. Press, 2004.

[13] I.C. Gohberg and M.G. Kreĭn, Introduction to the Theory of Linear Nonselfadjoint Operators, Transl. Math. Monographs 18, AMS, 1969.

[14] W. Groenevelt, Bilinear summation formulas from quantum algebra representations, Ramanujan J. 8 (2004), 383-416.

[15] D.P. Gupta, M.E.H. Ismail, D.R. Masson, Contiguous relations, basic hypergeometric functions and orthogonal polynomials. II Associated big q-Jacobi polynomials, J. Math. Anal. Appl. 171 (1992), 477-497.

[16] M.E.H. Ismail and D.R. Masson, q-Hermite polynomials, biorthogonal rational functions, and q-beta integrals, Trans. Amer. Math. Soc. 346 (1994), 63-116.

[17] R. Koekoek and R.F. Swarttouw, The Askey-scheme of hypergeometric orthogonal polynomials and its qanalogue, Tech. Report no. 98-17, TU Delft (1998), http://aw.twi.tudelft.nl/ koekoek/askey.html

[18] E. Koelink, One-parameter orthogonality relations for basic hypergeometric series, Indagationes Math. 14 (2003), 423-443.

[19] E. Koelink, Spectral theory and special functions, p. 45-84 in Laredo Lectures on Orthogonal Polynomials and Special Functions, (eds. R. Álvarez-Nodarse, F. Marcellán, W. Van Assche), Nova Sci. Publ., 2004.

[20] E. Koelink and J.V. Stokman, The Askey-Wilson function transform scheme, p. 221-241 in Special functions 2000: current perspective and future directions (eds. J. Bustoz, M.E.H. Ismail and S.K. Suslov), NATO Sci. Ser. II Math. Phys. Chem. 30, Kluwer, 2001.

[21] E. Koelink and J.V. Stokman, The big q-Jacobi function transform, Constr. Approx. 19 (2003), $191-235$.

[22] H.T. Koelink and J. Van der Jeugt, Convolutions for orthogonal polynomials from Lie and quantum algebra representations SIAM J. Math. Anal. 29 (1998), 794-822

[23] H. Rosengren, A new quantum algebraic interpretation of the Askey-Wilson polynomials, Contemp. Math. 254 (2000), 371-394.

California Institute of Technology, Mathematics 253-37, Pasadena, CA 91125, U.S.A.

E-mail address: stordal@caltech.edu

Technische Universiteit Delft, DiAM, PO Box 5031, 2600 GA Delft, the Netherlands

E-mail address: h.t.koelink@tudelft.nl 